Intensive care unit

\section{Critical care dysmotility: abnormal foregut motor function in the ICU/ITU patient}

\section{E M M Quigley}

While aspiration and feeding difficulties are well known challenges in the intensive care unit, their pathophysiology has been poorly understood. New information suggests that the critically ill are subject to profound alterations in motor and sensory function of the oesophagus, lower oesophageal sphincter, stomach, and duodenum which go some way to explaining their propensity to reflux and gastroparesis, with their attendant risks

A s medicine changes so should all aspects of medical care and knowledge; as more and more patients are subjected to surgical procedures of increasing complexity and risk and as survival rates from catastrophic illness rise, due to advances in surgery, anaesthesia, and intensive care, one would expect an associated growth in awareness of, and research into, the effects of critical illness on gut motor function. However, motor function and dysfunction, in this context, have received scant attention and have remained, for the most part, poorly understood. While a high prevalence of such phenomena as gastro-oesophageal reflux and gastroparesis are assumed, their true rates of occurrence have been scarcely documented. Similarly, while potential consequences of these entities, such as aspiration, oesophagitis, nosocomial pneumonia, feeding difficulties, and even gastric perforation are well known and, justifiably, feared, the effects of critical illness on oesophageal and gastric motor function have been little investigated. There are, it must be conceded, many reasons for this. Several factors, intrinsic to any intensive care (or therapy) unit (IC/TU) patient population, deter the clinician-scientist from embarking on the investigation of these subjects and confound the interpretation of any studies completed.

First and foremost, this is, by definition, a heterogeneous population. Depending on the nature of a particular unit, it may include among its patients a wide range of ages as well as a diverse catalogue of precipitating illnesses and events. Among the latter, prior surgery, raised intracranial pressure, intraabdominal or systemic sepsis, cranial, abdominal, or spinal trauma, and such conditions as acute pancreatitis and intestinal ischaemia will directly influence gut motor function. Within each of these categories further heterogeneity prevails; ileus following surgery being especially prevalent in relation not only to extensive abdominal procedures but also to abdominal aortic, cardiac, and spinal surgery. ${ }^{1}$ Pre-existing illnesses, such as diabetes, will also influence the response to acute stress and the likelihood of motor dysfunction. Electrolyte abnormalities are common in the critically ill and can influence the function of excitable tissues, including intestinal nerve and muscle.

Secondly, these patients are prey to a host of iatrogenic influences on gut motor function. The typical ICU patient receives significant sedation and analgesia, typically requires mechanical ventilation, is administered adrenergic agents for circulatory support, and relies on ether total parenteral (TPN) or enteral nutrition: all can influence foregut motor function. Administration of TPN, together with the metabolic effects of acute stress, may result in significant fluctuations in blood sugar levels with their attendant effects on the gut and, especially, gastric motility. Hyperglycaemia, for example, can profoundly suppress gastric motility and delay gastric emptying. ${ }^{2}$

Finally, these patients are critically ill and rarely fully conscious. Symptoms cannot be expressed, thereby ensuring that the consequences of motor dysfunction will be, for the most part, silent. These same factors may give rise to difficulties in relation to consent and ability to cooperate with research protocols.

Given this daunting background those who have endeavoured to answer crucial questions in this context are to be lauded and none more so than the Adelaide group which has recently presented us with two seminal works in this area. ${ }^{3}$ Taking advantage of the latest advances in technology, they have boldly gone where few have previously dared to tread and, in so doing, have provided us with some real progress in this area. Taken together, these studies provide some striking and clinically important observations on foregut motility in the critically ill. The first of these studies addressed oesophageal function, the second gastroduodenal; when combined they provide a frightening scenario which goes some way to explaining the prevalence of feeding intolerance and aspiration in this patient population.

Turning firstly to the study of oesophageal motor function. ${ }^{3}$ Among 15 mechanically ventilated patients they documented some striking abnormalities. Lower oesophageal sphincter (LOS) pressure was virtually non-existent (averaging just $2.2 \mathrm{~mm} \mathrm{Hg}$ !) and the motor response to reflux (secondary peristalsis) was strikingly impaired. Not surprisingly, reflux was frequent and often prolonged; in some patients, $\mathrm{pH}$ was below 4 for the entire six hour study period. The median percentage of time that $\mathrm{pH}$ was below 4 was $39 \%$ during fasting and $32 \%$ following nutrient administration. Reflux events were related either to absent LOS pressure or the occurrence of straining, on a background of marked LOS hypotension. Of considerable clinical relevance, most episodes of straining were precipitated by coughing, in turn usually provoked by endotracheal tube suction, a familiar scenario in any ICU. The ICU patient is clearly at extreme risk for acid reflux whose prevalence must have been greatly underestimated in the past if these data are in any way representative of the general ICU/ITU population. Acid may not be the only risk factor. This study incorporated motility, pH, and impedance measurements; the latter detecting many nonacid reflux events. In the ICU, parenteral acid suppression has become the norm; in this hypo- or achlorhydric environment bacterial overgrowth may occur in the stomach ${ }^{5}$ and could, through non-acid reflux events such as those described, lead to the development of aspiration pneumonia. It must be conceded that evidence for an increased incidence of nosocomial pneumonia in relation to acid suppression, in this context, has remained, for the most part, elusive. ${ }^{67}$ Nevertheless, given the findings of Nind and colleagues, ${ }^{3}$ one could speculate that therapy directed at the LOS or 


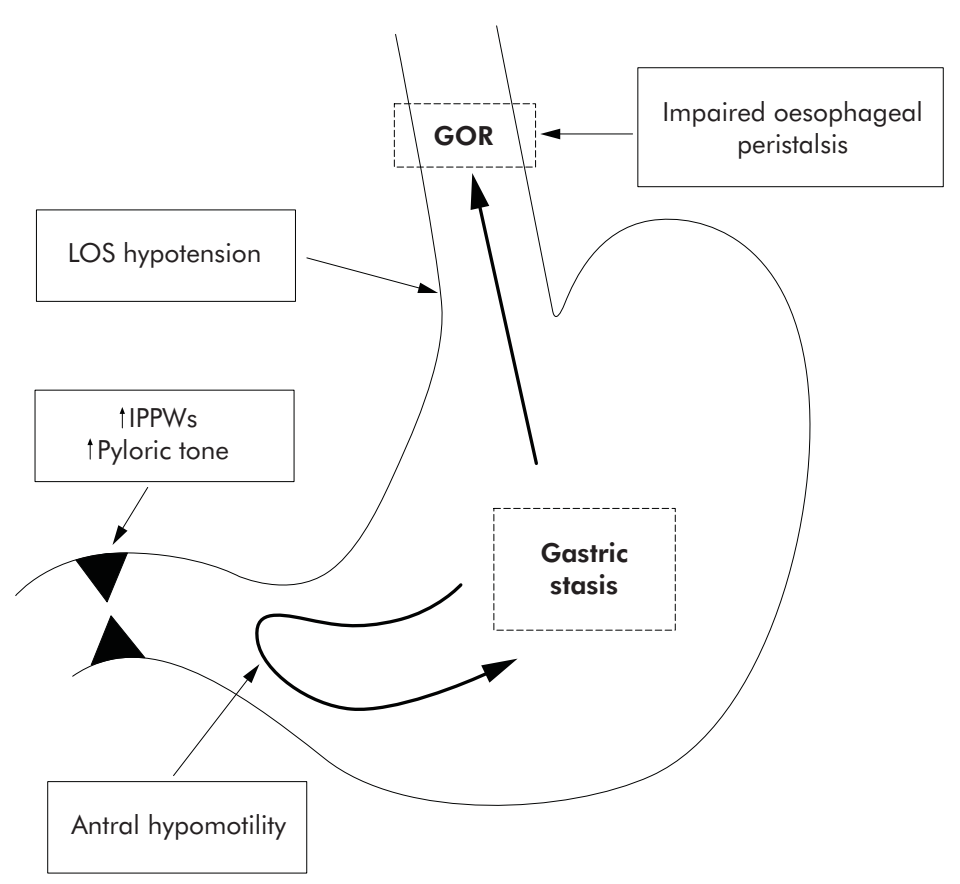

Figure 1 Abnormalities of oesophageal and gastric motor function in the intensive care unit patient. The "double jeopardy" of gastric and oesophageal motor dysfunction which conspire to promote reflux, aspiration, and feeding intolerance in the critically ill. IPPWs, isolated pyloric pressure waves; LOS, lower oesophageal sphincter, GOR, gastro-oesophageal reflux.

oesophageal body function may ultimately prove to be more appropriate than, or should be administered in addition to, acid suppression in the ICU.

In the second study, in this issue of $\mathrm{Gut}^{4}{ }^{4}$ efforts were exerted further "south", in the stomach and duodenum, but the findings were equally alarming (see page 1384). Fifteen mechanically ventilated ICU patients were subjected to antro-pyloro-duodenal manometry and gastric emptying in the fasted state and following intragastric and intraduodenal nutrient infusion at the very modest rate of $1 \mathrm{kcal} / \mathrm{min}$. The most striking finding was the profound inhibition of antral motility which resulted from either intragastric or intraduodenal nutrient installation; a similar rate of nutrient infusion to healthy volunteers had no effect on this parameter of gastric motor function. Intraduodenal nutrient also provoked isolated pyloric pressure waves and augmented pyloric tone, phenomena which would serve to further impair gastric emptying. Gastric emptying, was, not surprisingly, delayed. Taken together, these findings indicate a profound "upregulation" of the feedback loop which normally regulates the gastric emptying of nutrients and guarantees the steady delivery of $2-3 \mathrm{kcal} / \mathrm{min}$ to the small intestine, thus ensuring optimal digestive function. In the ICU patient, this very same mechanism appears to be preventing the egress of gastric contents in response to a duodenal nutrient delivery rate of just $1 \mathrm{kcal} / \mathrm{min}$, an observation that may go some way towards explaining the frequency of intolerance to enteral nutrition in this population. To add insult to injury, duodenal motility was disrupted with a failure to convert to a fed pattern and a persistence of burst activity, hardly the ideal accompaniment for the digestive process.

In both of these studies, the investigators were careful to control for some of the factors that have bedevilled prior investigations; nevertheless, the pathophysiology of these oesophageal and gastric motor changes remains unclear and the relative contributions of such factors as underlying illness, mechanical ventilation, or hypotension remain unresolved. The Chapman study, by invoking an abnormality in the intestinal sensing of nutrient, does indicate that, in terms of gastric function, the primary abnormality may not be simple "pump" failure, as has been described by others in the critically ill, ${ }^{8-10}$ but rather a hypersensitive intestino-gastric feedback loop. ${ }^{4}$

Regardless of the pathogenesis of these findings, everyone who cares for these patients must be aware of the triple jeopardy to which these patients are prone: LOS hypotension, oesophageal body dysmotility, and gastroparesis (fig 1). When combined, these factors expose the ICU patient to an extreme risk of aspiration of acid or, alternately, pathogen enriched, acid depleted gastric juice. These findings demand that we pay much more attention to motor dysfunction ${ }^{11}$ and the delivery of enteral nutrition to a highly vulnerable patient population. Here we are presented with a dramatic example of the need for ever greater emphasis on the "organic" manifestations of gastrointestinal myoneural pathology. ${ }^{12}$

Gut 2005;54:1351-1352.

doi: 10.1136/gut.2005.071027

Correspondence to: Professor E M M Quigley, Alimentary Pharmabiotic Centre, Department of Medicine, Clinical Sciences Building, Cork

University Hospital, Cork, Ireland;

e.quigley@ucc.ie

Conflict of interest: None declared.

\section{REFERENCES}

1 Quigley EMM. Acute intestinal pseudoobstruction. Curr Treat Options Gastroenterol 2000;3:273-86.

2 Rayner CK, Samsom M, Jones KL, et al. Relationship of upper gastrointestinal motor and sensory function with glycemic control. Diabetes Care $2001 ; 24: 371-81$.

3 Nind G, Chen W-H, Protheroe R, et al. Mechanisms of gastroesophageal reflux in critically ill mechanically ventilated patients. Gastroenterology 2005; 128:600-6.

4 Chapman M, Fraser R, Vozzo R, et al. Antropyloro-duodenal motor response to gastric and duodenal nutrient in critically ill patients. Gut 2005; 54:1384-90.

5 Williams C. Occurrence and significance of gastric colonization during acid-inhibitory therapy. Best Pract Res Clin Gastroenterol 2001;15:511-21.

6 Mallow S, Rebuck JA, Osler T, et al. Do proton pump inhibitors increase the incidence of nosocomial pneumonia and related infectious complications when compared to histamine-2 receptor antagonists in critically ill trauma patients? Curr Surg 2004;61:452-8.

7 Cook D, Guyatt G, Marshall J, et al. A comparison of sucralfate and ranitidine for the prevention of upper gastrointestinal bleeding in patients requiring mechanical ventilation. N Engl J Med 1998;338:791-7.

8 Heyland DK, Tougas G, King D, et al. Impaired gastric emptying in mechanically ventilated, critically ill patients. Intensive Care Med 1996;22:1339-44.

9 Kao CH, Chang Lai SP, Chieng PU, et al. Gastric emptying in head-injured patients. Am J Gastroenterol 1998;93:1 108-12.

10 Brand RE, DiBaise JK, Quigley EMM, et al. Gastroparesis as a cause of nausea and vomiting after high-dose chemotherapy and haemopoetic stem-cell transplantation. Lancet 1998;352:1985.

11 Quigley EMM. Gastrointestinal motility disorders in severely ill patients. Turk J Gastroenterol 2002; 13(suppl 2):39-44.

12 Quigley EMM. Enteric neuropathology-recent advances and implications for clinical practice. Gastroenterologist 1997;5:233-41. 
IBS

\section{Melatonin: a novel treatment for IBS?} S Elsenbruch

\section{Can poor sleep affect gastrointestinal symptoms in IBS? More on the "bad dreams cause bad bowels" hypothesis with reference to new treatment options}

$\mathrm{T}$ he issue of sleep in irritable bowel syndrome (IBS) is intriguing and relevant for several reasons. Firstly, complaints of poor sleep are extremely common in patients with IBS. In fact, self reported sleep disturbance can be regarded as one of the most important extraintestinal symptoms of IBS, which markedly affects quality of life and psychosocial well being. ${ }^{1}$ In spite of this, little is known about treatment options for this important extraintestinal symptom of IBS. Secondly, there is an overlap between IBS and fibromyalgia syndrome (FS), ${ }^{2}$ another pain syndrome which has also been linked to disturbed sleep physiology, ${ }^{3}$ as well as stress. ${ }^{4}$ If indeed a proportion of IBS patients share a common pathophysiological mechanism with FS, addressing the treatment of sleep related functions in patients with IBS would be a promising venue. Thirdly, hypervigilance has been discussed as one possible pathophysiological mechanism in IBS. $^{5}$ In this context, sleep studies using electroencephalogram techniques may be useful to reveal disturbed brain activity consistent with the hypothesis of hypervigilance/ altered arousal mechanisms in IBS. Finally, it has been suggested that reports of night time awakenings due to gastrointestinal symptoms, mainly abdominal or epigastric pain, may be used as a discriminatory factor between organic and functional disorder. ${ }^{6}$

A review of the existing literature confirms that a large percentage of IBS patients report sleep disturbances. ${ }^{7-14}$ Despite this overwhelming evidence of subjective sleep problems, it remains difficult to answer the question of whether these reports are based on objectively measurable abnormalities in sleep physiology. A total of eight studies have used objective methods to study sleep in IBS. ${ }^{910^{13} 15-18}$ Of those, three studies found no differences between IBS patients and controls on any polysomnographic measure and concluded that sleep architecture is normal in patients with IBS. ${ }^{9}{ }^{10} 17$ Only one study found what one might expect based on patients' subjective reports of insomniatype symptoms as well as disrupted, non-restful sleep-namely, markedly decreased slow wave sleep, higher arousal and awakening index, longer wake period after sleep onset, and more downward shifts to lighter sleep stages. ${ }^{7}$ A number of explanations exist for these heterogeneous results, including differences in patient populations in sex (that is, some have only included women), psychiatric comorbidities (some studies have excluded subjects with concurrent psychopathology, including depression and/or use of antidepressants), relatively small sample sizes, ${ }^{16-18}$ and inclusion of patients with sleep apnoea. ${ }^{18}$ Given this conflicting evidence, one could conclude that subjective sleep disturbances are not necessarily substantiated by objective sleep abnormalities in all IBS patients, although it is likely that subgroups of patients with objective sleep abnormalities exist. Determinants of subjective sleep problems in patients with IBS have been shown to include gastrointestinal symptom severity or profile $e^{1011} 19$ and psychological disturbances/distress. ${ }^{10} 1315$ In this context, it is of further interest to note that a large proportion of IBS patients do indeed report night time gastrointestinal symptoms, ${ }^{10}{ }^{11}$ even if they do not show polysomnographic evidence of sleep disruption. Therefore, the presence or absence of night time gastrointestinal symptoms is neither a valid discriminator between organic and functional disease ${ }^{6}$ nor a good indication of whether objective sleep abnormalities are in fact present.

Can bad sleep cause bad bowels or vice versa? This question, which ties into the relationship between sleep disturbance and severity of daytime gastrointestinal symptoms, remains difficult to answer. ${ }^{20}$ Sleep complaints have been found to be associated with perceived intensity of gastrointestinal symptoms. ${ }^{11}$ Using prospective diary assessments, two studies found that poor subjective sleep was associated with higher gastrointestinal symptoms the following day, ${ }^{81}$ lending support to the hypothesis that "bad sleep causes bad bowels" rather than vice versa. A more sophisticated way to address a possible cause-effect relationship between sleep disturbance and IBS is the experimental manipulation of sleep functions and subsequent study of effects on gastrointestinal symptomatology. This is what Song and colleagues ${ }^{22}$ have attempted, and the first randomised, double blind, placebo controlled trial on the effects of melatonin in IBS patients who reported sleep disturbances is described in this issue of Gut (see page 1402). ${ }^{22}$ Following two weeks of treatment, melatonin treated IBS patients demonstrated a significant reduction in reported abdominal pain, measured with an adapted version of the IBS symptoms evaluation score questionnaire, whereas no significant change was observed in the placebo group. In addition, rectal pressure and volume thresholds for both urgency and pain sensations were significantly increased following melatonin treatment. On the other hand, melatonin treatment had no effect on polysomnographic parameters, or measures of anxiety and depression.

In their study, the authors screened IBS patients for the presence of self reported sleep disturbance. ${ }^{22}$ Given the apparent inaccuracy of IBS patient reports concerning sleep disturbance and lack of consistent polysomnographic evidence of sleep abnormalities in IBS in the literature, it remains unclear what proportion of patients included in the trial did actually have manifest sleep problems. The lack of treatment effects on polysomnographic parameters in this study would lend support to the speculation that at least a proportion of the patient sample may not have had objective sleep abnormalities. Unfortunately, patients' basal polysomnographic data are difficult to evaluate in this respect as no healthy control group was included to show the extent to which a "first night effect" affected sleep at baseline. ${ }^{23}{ }^{24} \mathrm{~A}$ recent meta-analysis on the soporific effects of exogenous melatonin found that melatonin treatment significantly reduced sleep onset latency by 4.0 minutes, increased sleep efficiency by $2.2 \%$, and increased total sleep duration by 12.8 minutes. ${ }^{25}$ Given these modest effects, one may speculate that adaptation effects from the first to the second night (that is, improved sleep due to habituation) may have masked the effects of melatonin treatment. Future studies may consider screening of patients using polysomnography rather than questionnaires, which would have the further advantage of providing an adaptation night to minimise "first night effects", and make treatment induced changes in objective sleep parameters more easily detectable. 
Another issue that demands careful consideration when designing sleep (treatment) studies in IBS in the future is selection of patients with regard to psychiatric comorbidity. Song and colleagues $^{22}$ did not report screening for mood disorders in their patient population. However, mood disorders, especially anxiety and depression, are associated with alterations in sleep physiology, ${ }^{26}$ irrespective of the presence of other disorders such as IBS. Therefore, inclusion of IBS patients with mood disturbance can introduce difficulties regarding interpretation of sleep data, unless appropriate psychiatric control groups (for example, patients with depression but without IBS) are included. Psychological distress can affect the perception of sleep quality and daytime fatigue, and may modulate perception of other bodily symptoms (visceral and non-visceral) also. Anxiety and depression scores were not affected by melatonin treatment in the study by Song et al, and hence the mechanism by which melatonin treatment improved reported abdominal pain, and rectal pressure and volume thresholds for urgency and pain sensations remains unclear. Autonomic dysfunction has been implicated in the pathophysiology of IBS, including findings of autonomic disturbances during sleep in IBS patients. ${ }^{27}$ Based on evidence showing inhibitory effects of melatonin on the sympathetic nervous system in healthy humans, ${ }^{28} 29$ it is intriguing to speculate that modulation of autonomic functions may have played a role in the results reported by Song et al. Overall, these findings are intriguing and call for replication and further study.
Gut 2005;54:1353-1354

doi: $10.1136 /$ gut.2005.074377

Correspondence to: Department of Medical Psychology, University Clinic of Essen, Hufelandstr 55, 45122 Essen, Germany; sigrid.elsenbruch@uni-essen.de

Conflict of interest: None declared.

\section{REFERENCES}

1 Luscombe FA. Health-related quality of life and associated psychosocial factors in irritable bowel syndrome: a review. Qual Life Res 2000;9:161-76.

2 Chang $L$. The association of functional gastrointestinal disorders and fibromyalgia. Eur J Surg Suppl 1998;583:32-6.

3 Landis CA, Lentz MJ, Rothermel J, et al. Decreased sleep spindles and spindle activity in midlife women with fibromyalgia and pain. Sleep 2004:27:741-50.

4 Van Houdenhove B, Egle UT. Fibromyalgia: a stress disorder? Piecing the biopsychosocial puzzle together. Psychother Psychosom 2004;73:267-75

5 Berman SM, Naliboff BD, Chang L, et al. Enhanced preattentive central nervous system reactivity in irritable bowel syndrome. Am J Gastroenterol 2002;97:2791-7.

6 Lynn RB, Friedman LS. Irritable bowel syndrome. N Engl J Med 1993;329:1940-5.

7 Rotem AY, Sperber AD, Krugliak P, et al. Polysomnographic and actigraphic evidence of sleep fragmentation in patients with irritable bowel syndrome. Sleep 2003:26:747-52.

8 Jarrett M, Heitkemper M, Cain KC, et al. Sleep disturbance influences gastrointestinal symptoms in women with irritable bowel syndrome. Dig Dis Sci 2000;45:952-9

9 Elsenbruch S, Harnish MJ, Orr WC. Subjective and objective sleep quality in irritable bowel syndrome. Am J Gastroenterol 1999;94:2447-52.

10 Elsenbruch S, Thompson JJ, Hamish MJ, et al. Behavioral and physiological sleep characteristics in women with irritable bowel syndrome. Am J Gastroenterol 2002;97:2306-14.

11 Fass R, Fullerton S, Tung S, et al. Sleep disturbances in clinic patients with functional bowel disorders. Am J Gastroenterol 2000;95: $1195-200$

12 Whorwell PJ, McCallum M, Creed FH, et al. Noncolonic features of irritable bowel syndrome. Gut 1986;27:37-40

13 Heitkemper M, Charman AB, Shaver J, et al. Selfreport and polysomnographic measures of sleep in women with irritable bowel syndrome. Nurs Res 1998;47:270-7.

14 Zimmerman J. Extraintestinal symptoms in irritable bowel syndrome and inflammatory bowel diseases: nature, severity, and relationship to gastrointestinal symptoms. Dig Dis Sci 2003;48:743-9

15 Robert JJ, Orr WC, Elsenbruch S. Modulation of sleep quality and autonomic functioning by symptoms of depression in women with irritable bowel syndrome. Dig Dis Sci 2004;49:1250-8.

16 Orr WC, Crowell MD, Lin B, et al. Sleep and gastric function in irritable bowel syndrome: derailing the brain-gut axis. Gut 1997:41:390-3.

17 Gorard DA, Vesselinova-Jenkins CK, Libby GW, et al. Migrating motor complex and sleep in health and irritable bowel syndrome. Dig Dis Sci 1995:40:2383-9.

18 Kumar D, Thompson PD, Wingate DL, et al. Abnormal REM sleep in the irritable bowel syndrome. Gastroenterology 1992;103:12-17.

19 Schmulson M, Lee OY, Chang L, et al. Symptom differences in moderate to severe IBS patients based on predominant bowel habit. Am J Gastroenterol 1999;94:2929-35.

20 Orr WC. Sleep and functional bowel disorders: can bad bowels cause bad dreams? Am J Gastroenterol 2000:95:1118-21.

21 Goldsmith G, Levin JS. Effect of sleep quality on symptoms of irritable bowel syndrome. Dig Dis Sci 1993;38:1809-14.

22 Song GH, Leng PH, Gwee KA, et al. Melatonin improves abdominal pain in irritable bowel syndrome patients who have sleep disturbances: a randomised, double blind, placebo controlled study. Gut 2005;54:1402-7.

23 Agnew HW, Webb WB, Williams RL. The first night effect: an EEG study of sleep. Psychophysiology 1966;2:263-6.

24 Mendels J, Hawkins DR. Sleep laboratory adaptation in normal subjects and depressed patients ("first night effect"). Electroencephalogr Clin Neurophysiol 1967;22:556-8.

25 Brzezinski A, Vangel MG, Wurtman RJ, et al. Effects of exogenous melatonin on sleep: a metaanalysis. Sleep Med Rev 2005;9:41-50.

26 Benca RM, Okawa M, Uchiyama M, et al. Sleep and mood disorders. SleepMed Rev 1997; 1:45-56.

27 Orr WC, Elsenbruch S, Harnish MJ. Autonomic regulation of cardiac function during sleep in patients with irritable bowel syndrome. Am J Gastroenterol 2000;95:2865-71.

28 Ray CA. Melatonin attenuates the sympathetic nerve responses to orthostatic stress in humans. J Physiol 2003;551 (Pt 3): 1043-8.

29 Nishiyama K, Yasue H, Moriyama Y, et al. Acute effects of melatonin administration on cardiovascular autonomic regulation in healthy men. Am Heart J 2001;141:E9.

\section{The promise and perils of interpreting genetic associations in Crohn's disease}

\section{T T Trinh, J D Rioux}

\section{Extended analyses of inflammatory bowel disease susceptibility loci is advisable before definitive conclusions about their causative role can be drawn}

G enome wide linkage analysis has been an extremely successful method for mapping rare but highly penetrant genes in monogenic disorders. ${ }^{1}$ Its applications to common diseases have achieved limited success, however, due to the individually low heritability of each contributing gene. ${ }^{2}$ Alternatively, association based genetic studies, as described by Risch and Merikangas, ${ }^{3}$ can be powerful tools for identifying causal genes in common human diseases. ${ }^{14}$ To date, these methods have been used to follow up on linkage regions and to test for candidate genes and therefore have been limited by the previous linkage analyses or by the assumptions made regarding disease pathogenesis. Both of these factors can greatly diminish our ability to detect all possible causal variants contributing to complex disease traits. Over the last decade, however, technology and genetic resources have evolved dramatically. With the completion of the human 
genome sequence, recent development of high throughput genotyping technologies and knowledge of the patterns of genetic variation, it is now possible to perform genome wide association studies for common human diseases. Such genome wide approaches, although comprehensive, still face the analytical challenges of identifying true causal disease alleles. The prospects of identifying potentially significant associations must therefore be tempered by the perils of inaccurately drawn conclusions. Specifically, the progresses made in this new frontier will rely critically on proper execution and interpretation of genetic studies that are able to: (1) detect true positive from false positives; (2) distinguish causal variation from that which is in linkage disequilibrium (LD) (that is, cosegregation or non-random association of nearby alleles within a population); and (3) explain gene-function, gene-gene, and genotype-phenotype relationships.

The challenges presented by these three goals are nicely illustrated in the study of the genetic susceptibility of inflammatory bowel diseases (IBD). Despite the limitations of past genetic tools for complex diseases, the genetics of IBD has enjoyed a near unique situation of having successfully identified multiple associated alleles using these techniques. Several genome wide searches for IBD susceptibility loci had previously identified numerous genomic regions potentially containing IBD risk factors. Subsequently, association studies using positional mapping and candidate gene approaches further identified a few genetic regions that were independently replicated in various populations, including CARD $15^{5-10}$ and IBD5. ${ }^{11-16}$ However, the initial optimism is now being balanced with prudent realism. Difficulties such as replication of associations and translation of genetic association to causal functional consequences provide reason for cautious interpretation of results from genetic analyses of IBD and other complex diseases. The articles in this issue of Gut, by Török and colleagues ${ }^{17}$ in Germany and by Noble and colleagues ${ }^{18}$ in Scotland, highlight several of the present challenges with respect to two recently described IBD susceptibility regions (see pages 1416 and 1421). In the case of DLG5 (Drosophila discs large homologue 5), it is the challenge of replicating a putative positive association with IBD, and in the case of the organic cation transporter (OCTN) cluster in IBD5, it is the challenge of distinguishing the causal allele from those with which it is in LD.

In the case of DLG5, a susceptibility locus on chromosome 10 was initially described in 1999 by Hampe and colleagues, ${ }^{19}$ emerging from a genome wide linkage screen in a European cohort. Stoll et al further narrowed this risk region by using an association mapping approach and identified two distinct haplotypes in the region surrounding the DLG5 gene that were putatively associated with IBD and CD. ${ }^{20}$ Specifically, this group reported the association of a risk "haplotype D" (defined by a non-synonymous SNP $113 \mathrm{G} \rightarrow \mathrm{A}$ resulting in the amino acid substitution R30Q) with IBD and Crohn's disease (CD) in their family trios $\left(\chi^{2}=8.1, \quad p=0.004 ; \quad \chi^{2}=4.2\right.$, $\mathrm{p}=0.04$, respectively) with independent replication in their case control cohort $(p=0.0001$, odds ratio $(\mathrm{OR})=1.6) .^{20}$ They also described a protective "haplotype $\mathrm{A}^{\prime}$, identified by eight haplotype tagging single nucleotide polymorphisms (htSNPs), that was undertransmitted in the IBD trios and was further confirmed in an independent case control sample. Evidence for epistasis between DLG5 and CARD15 was also observed.

The initial excitement regarding DLG5 and IBD has now been met with some frustration, with the recent publication of variable findings from three other groups. Török and colleagues ${ }^{17}$ performed a replication study for DLG5 in an independent German case control group from Stoll and colleagues ${ }^{20}$ but could not confirm any association of the two previously reported haplotypes with IBD. They also did not find evidence of locus-locus interactions with CARD15. Along a similar line, Noble and colleagues ${ }^{18}$ also could not replicate the findings of Stoll et al in their Scottish population. ${ }^{20}$ They found no association of either haplotype with IBD, CD, or ulcerative colitis. Additionally, they could not confirm genetic epistasis between DLG5 and CARD15 in their population, nor did they identify specific phenotypic associations with DLG5.

In contrast, Daly and colleagues ${ }^{21}$ confirmed the association of DLG5 with IBD in two of their three European derived populations. Interestingly, they were able to replicate the association of IBD with the R30Q variant (haplotype D) in their Quebec/Italian case control cohort and in an independent Quebec/ UK family based study (approximate OR 1.25) but were not able to replicate it in a UK case control group. The authors suggested that the apparently inconsistent results from their own three studies were likely due to undetected phenotypic differences between the sample collections. In contrast, their three studies consistently demonstrated a lack of association to the putatively protective "haplotype A". They therefore attributed the original finding by Stoll et al as partially due to a statistical fluctuation and partially to a result of LD with the replicated R30Q association, as the presence of an overtransmitted allele or haplotype requires that the alternate allele or haplotype(s) have a net undertransmission (giving the appearance of a protective allele).

In general terms, the factors that are most likely to influence our ability to replicate true association findings are statistical power and consistency of phenotype across studies. The statistical power of an association study is dependent on sample size, frequency, and strength of the disease allele, as well as the frequency of the disease in the population. More specifically for the DLG5 variants, the prevalence of these alleles has not been extensively studied across ethnic or geographic subgroups; this information will help clarify whether differences observed among studies are truly effects of genetic or population heterogeneity, or rather an intrinsic weakness of modestly sized genetic studies due to sampling variation. The absence of $\mathrm{CD}$ associated CARD15 mutations and the IBD5 risk haplotype in the Japanese population supports the notion of variation of allelic frequencies in different populations. $^{22}{ }^{23}$ Alternatively, collection strategy and sampling bias must also be considered, as incomplete sampling can lead to overestimation of the frequency of some risk alleles and underestimation of others. ${ }^{24}$ The genetic effect (or penetrance) of the DLG5 variants is also not known although, as for most genes contributing to complex traits, the effects will likely be modest. Taking these factors into account, sample sizes of several thousand cases and controls will be needed to have $>90 \%$ power to replicate the findings of Stoll et al with confidence $(\mathrm{p}<0.01) .^{421}$

The current dilemma with DLG5 is not unique in the genetic elucidation of complex human diseases. An analogous situation was observed with type II diabetes, where PPARG was conclusively confirmed as a susceptibility locus only after examination of pooled data among studies, despite inconsistent findings among many small studies. ${ }^{25}$ Therefore, large collaborative efforts will be necessary to improve study power and enable adequately sized subpopulations to be studied for potential phenotypic associations with DLG5. Such large collaborative efforts will need to pay particular attention to phenotype, as this will not only be crucial for establishing the association of a genetic risk factor to disease but also for 
determining the relationship to clinical phenotype. Without a doubt, additional functional studies that provide convincing evidence of causality will be powerful adjuncts to any positive statistical findings. Finally, given the current status of the replication findings for DLG5, more work is required to define the exact nature of potential association to IBD.

In contrast with DLG5, the IBD5 risk haplotype has been firmly established as an IBD susceptibility region. Rioux et al first reported linkage for CD on chromosome $5 \mathrm{q} 31$ in a Canadian population with subsequent fine mapping of this locus to a $250 \mathrm{~kb}$ risk haplotype. ${ }^{11}{ }^{12}$ Several groups have independently confirmed this as a CD associated risk haplotype in different European and Caucasian populations. ${ }^{13-16}$ Unfortunately, identification of the underlying causal genetic variants within this region has been a more daunting task due to the strong LD across this region. ${ }^{11}$ In addition, specific phenotypic associations have not been clearly defined. Recently, a provocative study by Peltekova and colleagues ${ }^{26}$ proposed two causal SNPs in the carnitine/OCTN cluster located within the IBD5 risk haplotype that was associated with CD. The two mutations were in strong LD and created a two allele risk haplotype (TC haplotype) that was associated with CD independent of the extended IBD5 risk haplotype in their patient population. They provided preliminary functional studies demonstrating that these two SNPs resulted in impaired OCTN transporter function of various organic cations as well as carnitine, an essential cofactor in lipid metabolism. ${ }^{26} 27$ Based on the observed association of this TC haplotype with CD independent of the IBD5 extended haplotype, and a speculative link between OCTN function and intracellular homeostasis, they suggested that these two specific variants rather than other closely linked alleles were causal variants in $\mathrm{CD}$ susceptibility.

The present study by Török and colleagues $^{17}$ is the first published study that attempts to replicate the findings reported by Peltekova et al. Although they also found an association of the OCTN-TC haplotype with CD and an interaction with CD associated CARD15 mutations in their German case control cohort, they did not find conclusive genetic evidence that the OCTN polymorphisms were the likely causal variants. This was based on their observation that the association of the OCTN-TC haplotype with CD was not independent from another SNP (IGR2078a_1) also located within this tightly linke-d region that was chosen as a proxy for the extended IBD5 risk haplotype. The study highlights the difficulty in distinguishing a genetic variation that is causal from that which it is in LD (that is, are the OCTN genes or other unassayed SNPs in the IBD5 region the causal variants?). To firmly establish that the OCTN genes are the susceptibility genes within the IBD5 risk haplotype would require genetic evidence that OCTN-TC is not only independently associated with but also more strongly associated with IBD than the other genetic variants located within the extended haplotype on which it exists. This has not yet been convincingly demonstrated. In both of the above studies, the SNP IGR2078a_l was chosen as a proxy for the IBD $\overline{5}$ haplotype. Previous analysis of the IBD5 haplotype structure $^{11}$ indicates that this marker is located in a haplotype block at a significant distance from the block containing the OCTN1 and OCTN2 genes. A more relevant comparison to examine whether the OCTN variants are acting independently of the IBD5 haplotype would be to test other htSNPs within the same block. In addition, the relatively small numbers of samples in the two studies preclude a definitive answer to this question of independence of the OCTN variants and the other IBD5 haplotype variants. What are the factors that will influence studies seeking to address this question? In addition to the factors that affect our ability to replicate a true finding of association, the degree of recombination between the putative causal allele(s) and surrounding variants will determine our ability to distinguish the independence of the association signals.

Given the difficulties in resolving these dilemmas, future progress made with respect to OCTN and IBD5 will likely require supportive evidence from functional studies. Information providing a compelling biological explanation for how impairment of these OCTN genes leads to the clinical phenotypes in $C D$ would further strengthen any positive associations. Although Peltekova et al provide preliminary data linking the OCTN mutations with impaired cation transport, it is unclear how these defects would translate to an increased risk for intestinal inflammation. In fact, the OCTNs are widely expressed in various human tissues (that is, brain, intestine, skeletal muscle, heart, kidney, intestines), ${ }^{27}{ }^{29-31}$ and previously reported mutations in the human and mouse OCTN2 genes are associated with systemic carnitine deficiency, a condition characterised by diseases of skeletal muscles, cardiac muscles, and liver, rather than the intestinal system. ${ }^{27}{ }^{28}$ However, these two common variants in the OCTN genes may in the end turn out to be causative for IBD; much additional work will be necessary to prove causality and determine the precise mechanism of action.

Another apparent inconsistency in the literature regarding the IBD5 risk haplotype is its association with specific clinical phenotypes. Several groups have reported a lack of association between the IBD5 region and specific disease sites. ${ }^{11}{ }^{13-16}$ A UK group reported an association of the IBD5 haplotype with both perianal CD and ileal CD; however, on further analysis, they found that in fact the strongest association was for perianal CD with associated ileal disease ${ }^{16}$ Recently, Newman $e t$ al ${ }^{32}$ reported an association of the OCTN-TC haplotype with ileal $\mathrm{CD}$, independent of perianal disease involvement, which was further strengthened by the presence of CARD15 alleles. In this issue, Török and colleagues $^{17}$ report novel phenotypic associations with the IBD5/ OCTN-TC haplotype and colonic CD, and with non-fistulising and non-stricturing behaviour. This disparity among the literature reflects the inherent difficulties in genotype-phenotype correlation studies: small sample size of individual stratified subgroups and differences in phenotypic classification systems among studies. Future collaborative efforts to incorporate large data sets using standardised and rigorously defined phenotypic classification schemes will indeed be critical in clarifying these conflicting observations.

Genetic epistasis between both DLG5 and IBD5 with CARD15 was also extensively studied by these various groups, albeit with contrasting findings. This is not surprising, as interpretation of epistasis can be quite challenging. As discussed by Cordell, ${ }^{33}$ the same terminology has been used to apply to quite different definitions, as well as statistical and biological concepts. To add to this confusion is the dilemma in interpreting epistasis once it has been statistically identified. Statistical interaction does not necessarily imply interaction on a biological level. ${ }^{34}$ Until we can better define the correlation between statistical models and biological models, any interpretation of genetic epistasis should be made with caution.

In conclusion, the genetic revolution continues to progress with great momentum. This has been particularly evident in the field of IBD where the previously impossible task of identifying multiple causal genes has now become a reality. As we continue to generate interesting findings that have promising prospects in advancing our understanding of disease pathogenesis and ultimately, in the care of our patients, 
we must be aware of the perils and challenges that come with interpretation of the data. Keeping in mind these intermediate goals of finding true positive associations, identifying actual causal variants, and identifying true genegene and genotype-phenotype associations, we can guide our study design and data interpretation to ensure that our ultimate goal, furthering our knowledge of the genetics of complex diseases, is achieved.

\section{ACKNOWLEDGEMENTS}

JDR is supported by grants from the NIDDK and CCFA.

Gut 2005;54:1354-1357.

doi: $10.1136 /$ gut.2005.070920

\section{Authors' affiliations}

T T Trinh, The Broad Institute of MIT and Harvard, Cambridge, Massachusetts, USA and University of Virginia Health System, Digestive Health Center of Excellence, Charlottesville, Virginia, USA

J D Rioux, The Broad Institute of MIT and Harvard, Brigham and Women's Hospital, Department of Neurology, Harvard Medical School, Cambridge, Massachusetts, USA, and Université de Montréal, Montreal Heart Institute, Montréal, Québec, Canada

Correspondence to: Dr John D Rioux, 5000 Rue Bélanger, Montréal, Québec, Canada; rioux@broad.mit.edu

Conflict of interest: None declared.

\section{REFERENCES}

1 Hirschorn JN, Daly MJ. Genome-wide association studies for common diseases and complex traits. Nat Rev 2005;6:95-108.

2 Altmuller J, Palmer L, Fischer G. Genomewide scans of complex human diseases: true linkage is hard to find. Am J Hum Genet $2001 ; 69: 936-50$

3 Risch N, Merikangas K. The future of genetic studies of complex human diseases. Science 1996;273:1516-17.

4 Wang WYS, Barratt BJ, Clayton DG, et al. Genome-wide association studies: theoretical and practical concerns. Nat Rev 2005;6:109-18.
5 Hugot JP, Laurent-Puig P, Gower-Rousseau C, et al. Mapping of a susceptibility locus for Crohn's disease on chromosome 16. Nature 1996:379:821-3.

6 Hugot JP, Chamaillard M, Zouali $\mathrm{H}$, et al. Association of NOD2 leucine-rich repeat variants with susceptibility to Crohn's disease. Nature 2001;411:599-603.

7 Hampe J, Cuthbert A, Croucher PJ, et al. Association between insertion mutation in NOD2 gene and Crohn's disease in German and British populations. Lancet 2001;357:1925-8.

8 Hampe J, Grebe J, Nikolaus S, et al. Association of NOD2 (CARD15) genotype with clinical course of Crohn's disease: a cohort study. Lancet 2002;359:1661-5.

9 Cavanaugh J. International collaboration provides convincing linkage replication in complex disease through analysis of a large pooled data set: Crohn's disease and chromosome 16. Am J Hum Genet 2001;68:1165-71

10 Ogura Y, Bonen DK, Inohara N, et al. A frameshift mutation in NOD2 associated with susceptibility to Crohn's disease. Nature 2001;411:603-6.

11 Rioux JD, Daly MJ, Silverberg MS, et al. Genetic variation in the $5 q 31$ cytokine gene cluster confers susceptibility to Crohn disease. Nat Genet $2001 ; 29: 223-8$.

12 Rioux JD, Silverberg MS, Daly MJ, et al. Genomewide search in Canadian families with inflammatory bowel disease reveals two novel susceptibility loci. Am J Hum Genet 2000;66:1863-70.

13 Mirza MM, Fisher S, King K, et al. Genetic evidence for interaction of the $5 \mathrm{q} 31$ cytokine locus and the CARD1 5 gene in Crohn disease. Am J Hum Genet 2003;72:1018-22.

14 Giallourakis C, Stoll M, Miller K, et al. IBD5 is a general risk factor for inflammatory bowel disease: replication of association with Crohn disease and identification of a novel association with ulcerative colitis. Am J Hum Genet 2003;73:205-11.

15 Negoro K, McGovern DPB, Kinouchi Y, et al. Analysis of the IBD5 locus and potential genegene interactions in Crohn's disease. Gut 2003;52:541-6.

16 Armuzzi A, Ahmad T, Ling K-L, et al. Genotypephenotype analysis of the Crohn's disease susceptibility haplotype on chromosome $5 q 31$. Gut 2003;52:1133-9.

17 Török H-P, Glas J, Tonenchi L, et al. Polymorphisms in the DLG5 and OCTN cation transporter genes in Crohn's disease. Gut 2005;54:1421-7.

18 Noble CL, Nimmo ER, Drummond H, et al. DLG5 variants do not influence susceptibility to inflammatory bowel disease in the Scottish population. Gut 2005;54:1416-20.

19 Hampe J, Schreiber S, Shaw SH, et al. A genomewide analysis provides evidence for novel linkages in inflammatory bowel disease in a large European cohort. Am J Hum Genet 1999;64:808-16.

20 Stoll M, Corneliussen B, Costello CM. Genetic variation in DLG5 is associated with inflammatory bowel disease. Nat Genet 2004;36:476-80.

21 Daly MJ, Pearce AV, Farwell L. Association of DLG5 R30Q variant with inflammatory bowel disease. Eur J Hum Gen 2005; 13:835-9.

22 Inoue N, Tamura K, Kinouchi Y. Lack of common NOD2 variants in Japanese patients with Crohn's disease. Gastroenterol 2002; 123:86-91.

23 Yamazaki K, Takazoe M, Tanaka T. Absence of mutation in the NOD2/CARD15 gene among 483 Japanese patients with Crohn's disease. $J$ Hum Genet 2002;47:469-72.

24 Williams CN, Kocher K, Lander ES, et al. Using a genome-wide scan and meta-analysis to identify a novel IBD locus and confirm previously identified IBD loci. Inflamm Bowel Dis 2002;8:375-80

25 Atshuler D, Hirschorn JN, Klannemark M. The common PPARg Prol 2Ala polymorphisms is associated with decreased risk of type 2 diabetes. Nat Genet 2000;26:76-80.

26 Peltekova VD, Wintle RF, Rubin LA, et al. Functional variants of OCTN cation transporter genes are associated with Crohn disease. Nat Genet 2004;36:471-5

27 Lahjouji K, Mitchell GA, Qureshi IA. Carnitine transport by organic cation transporters and systemic carnitiine deficiency. Mol Genet Metab 2001;73:287-97.

28 Tamai I, Ohashi R, Nezu Jl, et al. Molecular and functional characterization of organci cation/ carnitiine transporter family in mice. J Biol Chem 2000;275:40064-72.

29 Kekuda R, Prasad PD, Wu X. Cloning and functional characterization of a potential sensitive polyspecific organic cation transporter (OCT3) most abundantly expressed in placenta. J Biol Chem 1998:273:15971-9.

30 Tamai I, Yabuuchi H, Nezu J. Cloning and characterization of a novel human pH-dependent organic cation transporter, OCTN1. FFBS Lett 1997:419:107-11.

31 Wu X, Prasad PD, Leibach FH. cDNA sequence, transport function, and genomic organization of human OCTN2, a new member of the organic cation transporter family. Biochem Biophys Res Commun 1998;246:589-95.

32 Newman B, Gu X, Wintle RF, et al. A risk haplotype in the solute carrier family 22A4/22A5 gene cluster influences phenotypic expression of Crohn's disease. Gastroenterol 2005; 128:260-9.

33 Cordell HJ. Epistasis: what it means, what it doesn't mean, and statistical methods to detect it in humans. Hum Mol Gene 2002; 11:2463-8.

34 Witte J. Gene-environmental interaction. In: Armitage P, Colton T, eds. Encyclopedia of biostatistics. Chichester: Wiley, 1998:1613-14. 


\section{The importance of keeping in touch: regulation of cell-cell contact in the exocrine pancreas}

\section{V Apte, J S Wilson}

\section{New insights into the mechanisms regulating acinar cell-cell contact in the exocrine pancreas, with evidence to support a role for PTPк as a key molecule in stabilisation of the adhesion complex in acinar cells via continuous dephosphorylation of the cadherin-catenin complex}

$\mathrm{T}$ he formation of tissues and organs of multicellular organisms during embryonic development involves a highly regulated process of integration and segregation of heterogeneous cell populations into organised cell patterns. ${ }^{1}$ One of the major regulators of the processes of cell migration, proliferation, and differentiation during organogenesis is cell-cell adhesion, which is predominantly mediated by cell surface glycoproteins. These cell adhesion proteins can respond to cell signalling events and can also transduce signals into the cell. ${ }^{2}$ Four major groups of cell adhesion proteins have been described, including integrins, immunoglobulins, selectins, and cadherins.

Cadherins are a superfamily of integral membrane proteins that are subdivided into six gene families. ${ }^{1}$ E-cadherin, a member of the classical cadherin type I family, has been widely studied in a variety of epithelial cell systems..$^{2-5}$ Like most cadherins, E-cadherin is a transmembrane glycoprotein with an extracellular domain that interacts with the extracellular domains of cadherin molecules of adjacent cells in a calcium dependent homophilic manner. The highly conserved intracellular (cytoplasmic) domain of E-cadherin functions as a binding site for catenins (cytoplasmic proteins that anchor E-cadherin to the cell cytoskeleton). Typically, E-cadherin binds to $\beta$-catenin or pl20 catenin in the cells; these catenins in turn are associated with $\alpha$-catenin which links the cadherincatenin complex to the cytoskeletal protein F-actin within the cell. The formation of cadherin-catenin complexes is critical to cell-cell adhesion. Both cadherins and catenins contain phosphorylation sites which regulate their function at intercellular junctions. ${ }^{6}$ It has now been established that cell adhesion complexes do not function as static structures but are subjected to highly regulated changes during tissue development and/or repair. ${ }^{7}$

Loss or alteration of cell-cell contact is a feature of many pathological states, including the relatively slow process of tumour invasion and metastasis and the relatively rapid events of inflammation and oedema formation. Regulation of adhesion complexes in tumorigenesis and inflammatory conditions of the skin has been widely studied ${ }^{8-10}$ but little is known about the factors regulating cellcell contact in a complex epithelial organ such as the pancreas. The functional unit of the exocrine pancreas is the acinus, comprising individual acinar cells arranged around a central lumen. ${ }^{11}$ Each acinar cell is in close contact with adjacent acinar cells through cell-cell adhesions, including tight junctions (which seal the paracellular routes between adjacent cells), GAP junctions (intercellular channels connecting the cytoplasm of adjacent cells), and adherens junctions (specialised regions of adhesion at the basolateral plasma membrane). Acute inflammation of the pancreas (acute pancreatitis) is characterised by the development of interstitial oedema (associated with loss of contact between acinar cells within the tissue ${ }^{12}$ ) and infiltration of inflammatory cells into the parenchyma.

The study by Schnekenburger and colleagues $^{13}$ in this issue of Gut deals with the regulation of adherens junction integrity in pancreatitis and represents a logical progression of previous work by this group ${ }^{14}{ }^{15}$ describing the dissociation, internalisation, and reassembly of the adherens junction during acute experimental pancreatitis (see page 1445). In the current study, ${ }^{13}$ the authors have endeavoured to identify the mechanisms responsible for adherens junction dissociation in pancreatitis using both in vitro (isolated acini exposed to supramaximal caerulein stimulation) and in vivo (mouse model of mild caerulein induced pancreatitis) approaches. The self limiting nature of pancreatic injury in the caerulein pancreatitis model enabled the authors to study cell adhesion regulation not only during injury but also during the restitution (repair) phase.

Cell-cell contact can potentially be disturbed by alterations in the expression or function of adherens junction proteins and/or by disruptions in the actin cytoskeleton. A number of recent reports in the literature (using cancer cell lines, metastatic fibroblasts, epithelial cells) have described a role for tyrosine phosphorylation of adherens junction proteins in the perturbation of cell-cell contacts. ${ }^{4-18}$ Corollary evidence has been provided by studies demonstrating that a dephosphorylated state of cell adhesion proteins (via the action of protein tyrosine phosphatases (PTPs) ) is essential for the maintenance of an intact cell adhesion complex. ${ }^{19} 20$

Using a number of elegant experimental protocols, Schnekenburger and colleagues ${ }^{13}$ have been able to describe a time course of the changes occurring in the adhesion complexes of acinar cells during the development (at one hour after caerulein injection) and resorption (at 48 hours after caerulein injection) of oedema in acute pancreatitis. A significant increase in tyrosine phosphorylation of catenins ( $\beta$-catenin and pl20 catenin) and E-cadherin was observed early in the course of injury (at one and two hours after caerulein injection), although expression of the proteins themselves remained unchanged. The increase in tyrosine phosphorylation of $\beta$-catenin and E-cadherin coincided with dissociation of the complex from the transmembrane phosphatase РТРК (known to be constitutively associated with the cadherin-catenin complex in normal cells), redistribution of adhesion proteins from the lateral and apical cell membrane to the cytosol (internalisation), and subsequently, an association of the complex with the cytosolic protein tyrosine phosphatase PTP SHP-1 over 2-4 hours. At 48 hours, resorption of oedema was associated with the reassembly of the adhesion complex and its relocation to the lateral and apical cell membranes. In vitro studies with isolated acini demonstrated that inhibition of PTPs by orthovanadate induced acinar cell dissociation in a manner similar to that observed with supramaximal caerulein, supporting the concept that tyrosine phosphorylation was a key regulator of the cadherincatenin complex.

Two observations of interest in this study were: (i) the lack of any changes 
in protein expression of the adhesion complex during caerulein pancreatitis in vivo and (ii) the in vitro finding that disruption of the actin cytoskeleton did not influence cell dissociation. Both of these observations strengthen the concept that pancreatic acinar cell dissociation during caerulein pancreatitis is predominantly regulated by tyrosine phosphorylation and dephosphorylation of the proteins of the adhesion complex, and is independent of any alterations in protein levels or the integrity of the actin cytoskeleton.

This study makes a significant contribution to our understanding of the mechanisms regulating acinar cell-cell contact in the exocrine pancreas, with evidence to support a role for РТРк as a key molecule in stabilisation of the adhesion complex in acinar cells via continuous dephosphorylation of the cadherin-catenin complex. However, important questions remain with respect to disruption of acinar contact in the pathophysiological setting. Schnekenburger et al ${ }^{13}$ assert that acinar dissociation in caerulein pancreatitis is observed ultrastructurally before the development of oedema, thereby excluding a "physical" cause (such as increased pressure due to accumulation of fluid in the interstitium) for acinar cell dissociation. If acinar dissociation precedes (and is not the effect of) oedema, it would be logical to ask what factors disrupt the mechanisms regulating cell-cell adhesion in the earliest stages of pancreatitis and what factors stimulate reassembly of disrupted adhesion complexes during the repair phase of the disease. Future studies in this area will no doubt be designed to address these issues by examining: (i) the cellular events during acute pancreatitis which disrupt the constitutive association of РТРК with the adhesion complex and/or trigger tyrosine phosphorylation of the complex; (ii) whether proteolytic cleavage of E-cadherin during acute necroinflammation plays a role in triggering tyrosine phosphorylation of the adhesion complex leading to cell dissociation and; and (iii) the potential roles of intracellular catenins in restitution of the adhesion complex after internalisation. Elucidation of the above processes has the potential to identify specific molecules/pathways that could be therapeutically targeted to: (i) prevent/inhibit disruption of cell adhesion in the initial phase of acute pancreatitis (thereby maintaining a physical barrier to inhibit inflammatory cell infiltration into the gland) and (ii) stimulate the reassembly of the cell adhesion complex during recovery from the disease.

Gut 2005;54:1358-1359.

doi: 10.1136/gut.2005.070953

\section{Authors' affiliations}

M V Apte, J S Wilson, Pancreatic Research Group, The University of New South Wales, Sydney, Australia

Correspondence to: Associate Professor MApte, Director, Pancreatic Research Group, South Western Sydney Clinical School, The University of New South Wales, Level 2, Thomas and Rachel Moore Education Centre, Liverpool Hospital, Liverpool, NSW 2170, Australia; m.apte@unsw.edu.au

Conflict of interest: None declared.

\section{REFERENCES}

1 Aberle H, Schwartz H, Kemler R. Cadherincatenin complex: Protein interactions and their implications for cadherin function. J Cell Biochem 1996;61:514-23.

2 Gooding JM, Yap KL, Ikura M. The cadherincatenin complex as a focal point of cell adhesion and signalling: New insights from threedimensional structures. Bioessays 2004;26:497-51 1

3 Behrens J. Cadherins and catenins: Role in signal transduction and tumor progression. Cancer Metastasis Rev 1999;18:15-30.

4 Perez-Moreno M, Jamora C, Fuchs E. Sticky business: Orchestrating cellular signals at adherens junctions. Cell 2003;112:535-48.
5 Zbar AP, Simopoulos C, Karayiannakis AJ. Cadherins: An integral role in inflammatory bowel disease and mucosal restitution. J Gastroenterol 2004:39:413-21.

6 Grunwald GB. The structural and functional analysis of cadherin calcium-dependent cell adhesion molecules. Curr Opin Cell Biol 1993;5:797-805.

7 Gumbiner BM. Regulation of cadherin adhesive activity. J Cell Biol 2000;148:399-404.

8 Guilford P, Hopkins J, Harraway J, et al. Ecadherin germline mutations in familial gastric cancer. Nature 1998;392:402-5.

9 Perl AK, Wilgenbus P, Dahl U, et al. A causal role for E-cadherin in the transition from adenoma to carcinoma. Nature 1998;392:190-3.

10 Payne AS, Hanakawa Y, Amagai M, et al Desmosomes and disease: Pemphigus and bullous impetigo. Curr Opin Cell Biol 2004; 16:536-43.

11 Bockman DE. Anatomy of the pancreas. In: Go VLW, Dimagno EP, Gardner JD, et al. The pancreas: biology, pathobiology and disease. New York: Raven Press, 1993:1-8.

12 Steer ML. Etiology and pathophysiology of pancreatitis. In:Go VLW, Dimagno EP, Gardner JD, et al.,eds. The pancreas: biology, pathobiology and disease. New York: Raven Press, 1993:581-91.

13 Schnekenburger J, Mayerle J, Krüger B, et al. Protein tyrosine phosphatase $\kappa$ and SHP- 1 are involved in the regulation of cell-cell contacts at adherens junctions in the exocrine pancreas. Gut 2005; 54: 1445-55.

14 Lerch MM, Lutz MP, Weidenbach $\mathrm{H}$, et al. Dissociation and reassembly of adherens junctions during experimental acute pancreatitis. Gastroenterology 1997;113:1355-66.

15 Schnekenburger J, Mayerle J, Simon P, et al. Protein tyrosine dephosphorylation and the maintenance of cell adhesions in the pancreas. Ann N Y Acad Sci 1999:880:157-65.

16 Matsuyoshi N, Hamaguchi M, Taniguchi S, et al. Cadherin-mediated cell-cell adhesion is perturbed by $\mathrm{v}$-src tyrosine phosphorylation in metastatic fibroblasts. J Cell Biol 1992;118:703-14.

17 Behrens J, Vakaet L, Friis R, et al. Loss of epithelial differentiation and gain of invasiveness correlates with tyrosine phosphorylation of the E-cadherin/ beta-catenin complex in cells transformed with a temperature-sensitive v-src gene. J Cell Biol 1993; 120:757-66

18 Calautti E, Cabodi S, Stein PL, et al. Tyrosine phosphorylation and src family kinases control keratinocyte cell-cell adhesion. J Cell Biol 1998;141:1449-65.

19 Zondag GC, Moolenaar WH. Receptor protein tyrosine phosphatases: Involvement in cell-cell interaction and signaling. Biochimie 1997;79:477-83.

20 Muller T, Choidas A, Reichmann E, et al. Phosphorylation and free pool of beta-catenin are regulated by tyrosine kinases and tyrosine phosphatases during epithelial cell migration. J Biol Chem 1999;274:10173-83.

\section{Sir Francis Avery Jones BSG Research Award 2005}

Applications are invited by the Education Committee of the British Society of Gastroenterology who will recommend to Council the recipient of the 2006 Award. Applications (20 copies) should include:

- A manuscript (2 A4 pages only) describing work conducted

- A bibliography of relevant personal publications

- An outline of the proposed content of the lecture, including title

- A written statement confirming that all or a substantial part of the work has been personally conducted in the UK or Eire.

Entrants must be 40 years or less on 31 December 2005 but need not be a member of the Society. The recipient will be required to deliver a 30 minute lecture at the annual meeting of the Society in Birmingham in March 2006. Applications (20 copies) should be made to the Honorary Secretary, British Society of Gastroenterology, 3 St Andrews Place, London, NW1 4LB by 1 December 2005. 
TB in patients with Crohn's disease

\section{Preventing TB in patients with Crohn's disease needing infliximab or other anti-TNF therapy}

D S Rampton

In patients with Crohn's disease, the increased risk of active tuberculosis (TB) associated with infliximab makes it necessary to screen for active and latent TB before this or other anti-tumour necrosis factor treatment is begun. This paper outlines how such screening should be undertaken, and how to decide which patients need antituberculous treatment or chemoprophylaxis before infliximab.

\section{SUMMARY}

The increased risk of active tuberculosis (TB) associated with infliximab makes necessary a screen for active and latent TB before this or other anti-tumour necrosis factor (TNF) treatment is begun in patients with Crohn's disease. This paper outlines how such screening should be undertaken, and how to decide which patients need antituberculous treatment or chemoprophylaxis before infliximab. All patients need a careful history for TB and a chest $x$ ray. The minority of patients with a history of TB or an abnormal chest $x$ ray should be referred for assessment by a TB specialist. Of the remainder, those with Crohn's disease who are on immunosuppressive therapy do not require tuberculin testing. Comparison of their risk of TB while on anti-TNF therapy with the risks of chemoprophylaxis induced hepatitis indicates that black Africans aged over 15 years, South Asians born outside the UK, and other ethnic groups resident in the UK for less than five years should be considered for chemoprophylaxis with isoniazid for six months. For how to minimise the risk of $\mathrm{TB}$ in the small minority of patients with inflammatory bowel disease not on immunosuppressive treatment, readers are referred to the more detailed guidelines published in Thorax.

\section{INTRODUCTION}

Infliximab is of proven benefit in the treatment of chronic active Crohn's disease $^{2}$ as well as in rheumatoid arthritis $^{3}$ and ankylosing spondylitis; ; preliminary data suggest it may also have a therapeutic role in refractory active ulcerative colitis. ${ }^{5-7}$ An increased risk of tuberculosis (TB) was noted soon after the introduction of infliximab in the late 1990s. To address the question of how best to prevent $\mathrm{TB}$ in patients needing infliximab and other anti-tumour necrosis factor (TNF) therapies, the British Thoracic Society Standards of Care Committee formed a subcommittee chaired by Professor Peter Ormerod and comprising also representatives of the British Society of Rheumatology and British Society of Gastroenterology (DSR). The full recommendations of this group, and their rationale, are reported in Thorax ${ }^{1}$ (also online at http:// thorax.bmjjournals.com/cgi/rapidpdf/thx. 2005.046797vl). This brief article aims to bring to the attention of gastroenterologists the principal conclusions as they relate specifically to the management of patients with Crohn's disease; to facilitate this aim, an algorithm which outlines the approach to minimising the risk of $\mathrm{TB}$ is provided (fig 1).

\section{WHAT IS THE RISK OF TB?}

In the general population in the UK, the incidence of TB depends on a range of factors which include age, ethnicity, and country of birth. ${ }^{8}$ The annual risk of TB in the UK is increased at least 30 -fold in Black Africans aged over 15 years and in South Asians born outside the UK; it is even greater in people from other ethnic groups resident in the UK for less than five years.

In Crohn's disease which has not been treated with infliximab, the incidence of TB is unknown; indeed, in some patients it may of course be difficult, initially at least, to distinguish the one diagnosis from the other. Infliximab appears to increase the background risk of $\mathrm{TB}$ by approximately fivefold in both Crohn's disease and rheumatoid arthritis, ${ }^{1}$ most, although not all, cases being extrapulmonary and occurring within the first three months of treatment. ${ }^{9-13}$ Although the incidence of infliximab related TB may now be falling due to improved risk assessment, chemoprophylaxis (see below), and/or reporting fatigue, ${ }^{11}$ complacency is clearly inappropriate: mortality of $\mathrm{TB}$ in the early days of its recognition in association with the use of infliximab approached $10 \%$.

\section{HOW CAN THE RISK OF TB BE MINIMISED IN PATIENTS TO BE GIVEN INFLIXIMAB (FIG 1)?}

Recommendations from several sources, including the European Agency for Evaluation of Medicinal Products (EMEA) and the National Institute for Clinical Excellence (NICE) (see below), agree that patients in whom the use of anti-TNF therapy is being considered should be meticulously questioned about prior TB and its treatment, and have a chest $x$ ray taken. ${ }^{1{ }^{12-16}}$

\section{Patients with a history of TB and/or} abnormal chest $x$ ray

Patients with a history of $\mathrm{TB}$ or an abnormal chest $x$ ray should be referred directly to a specialist with expertise in TB. ${ }^{1}$ Those with active TB should receive standard antituberculous chemotherapy for at least two months before starting on infliximab. Patients with a chest $x$ ray showing previous $\mathrm{TB}$, or with a history of previous extrapulmonary TB which has been fully treated, should be carefully monitored during infliximab therapy; those in whom treatment may have been inadequate should have active TB excluded by appropriate investigation and should be started on chemoprophylaxis two months before starting infliximab.

\section{Patients with no history of TB and} normal chest $x$ ray

Some guidelines have suggested that a tuberculin test should be used to direct the optimal approach in this group of patients. $^{212}{ }^{13}$ Recent data however have confirmed a very high incidence of anergy in patients with Crohn's, ${ }^{14}$ and the EMEA recommendations specifically warn prescribers of the risk of false negative skin test results in severely ill or immunocompromised patients with Crohn's disease ${ }^{15}$. Indeed, since under existing (2002) NICE guidelines ${ }^{16}$ all patients with Crohn's disease in the UK needing infliximab will be chronically ill and currently or recently taking corticosteroids and/or immunomodulatory drugs, tuberculin testing will not assist in decision making and is considered unnecessary. ${ }^{1}$ (There are no data on the incidence of anergy to tuberculin 


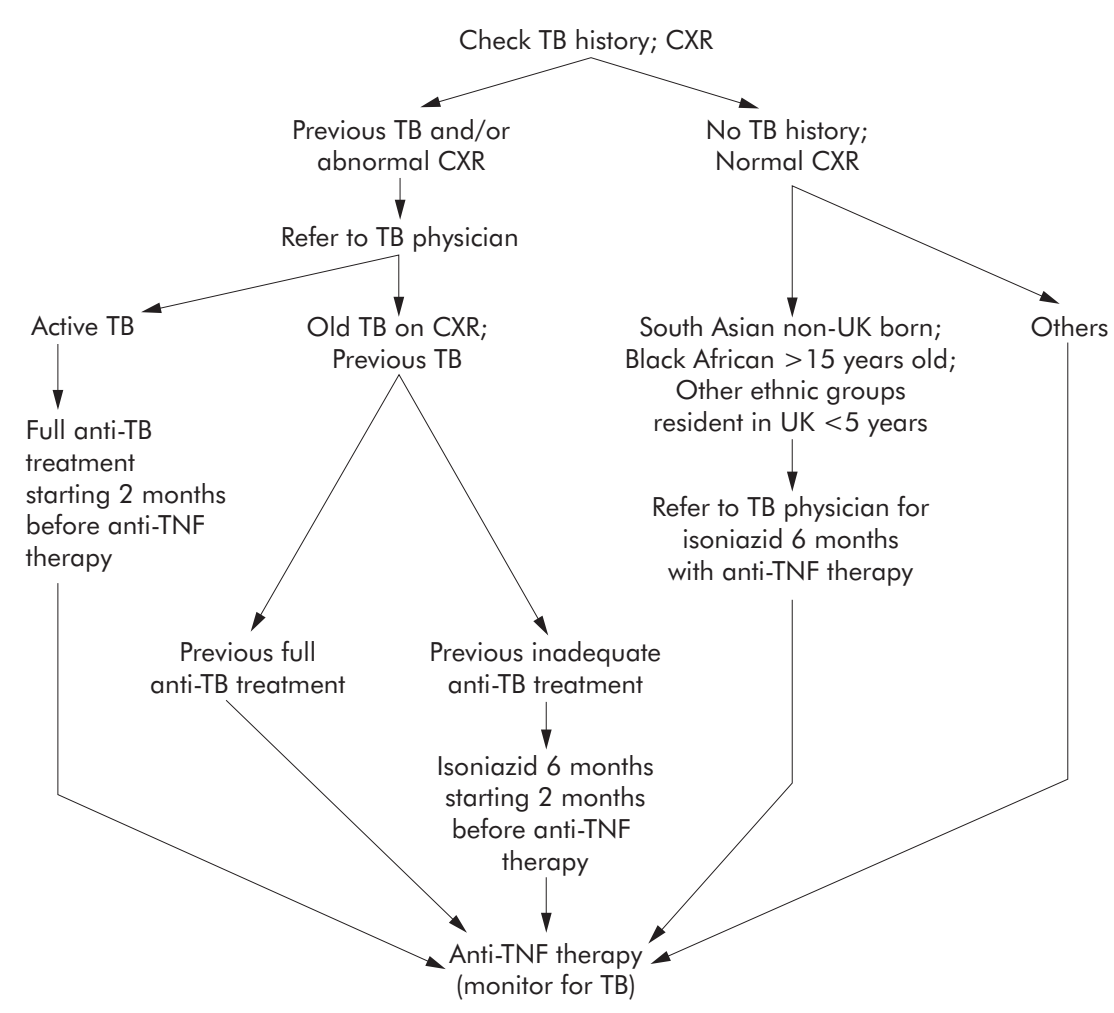

Figure 1 Algorithm to indicate the approach to prevention of tuberculosis (TB) in patients on immunosuppressants who need infliximab or other anti-tumour necrosis factor (TNF)- $\alpha$ therapy for Crohn's disease. The high incidence of anergy in patients with Crohn's disease who take immunosuppressants ${ }^{14}{ }_{15}$ makes tuberculin skin testing unreliable and unnecessary. The decision about TB chemoprophylaxis in individual patients with no history of TB and a normal chest $x$ ray (CXR) is dependent on a comparison of their ethnicity related risk of acquiring TB during anti-TNF therapy and the risk of drug induced hepatitis during chemoprophylaxis (see text and British Thoracic Society'). (For recommendations about the prevention of TB in the small minority of patients with Crohn's disease not taking concomitant immunosuppressive therapy, and in those with ulcerative colitis in whom infliximab is being considered, see British Thoracic Society').

in patients with ulcerative colitis; in these, currently exceptional patients, the guidelines described in full by the British Thoracic Society ${ }^{1}$ should be followed.)

What does need to be considered is the annual risk of $\mathrm{TB}$ in individual patients to be given infliximab: as indicated above, this is increased about fivefold by infliximab and still further in some ethnic groups. This risk needs to be balanced against the risk of side effects caused by TB chemoprophylaxis, which is dependent on the regimen to be used. ${ }^{1}$ The commonest regimen, isoniazid for six months, has a hepatitis risk rate of about 280/100 000 treated patients. ${ }^{1}$ Two shorter regimens, rifampicin with isoniazid for three months and rifampicin with pyrazinamide for two months, cause serious hepatitis much more often (1800 and 6600/ 100000 treated patients, respectively). ${ }^{1}$

These considerations mean that, in general, Caucasians in the UK with no history of TB and a normal chest $x$ ray need no TB chemoprophylaxis. In contrast, even if they have no TB history, and their chest $x$ ray is normal, Black complications of the use of infliximab In each patient in whom therapy with infliximab is being considered, a plan should be drawn up based on their history, chest $x$ ray, ethnicity, place of birth, and duration of residence in the UK (see fig 1). Implementation of these recommendations is likely to reduce dramatically the risk of $\mathrm{TB}$ in patients given infliximab and other anti-TNF agents.

\section{ACKNOWLEDGEMENTS}

I am grateful to Professor P Ormerod (British Thoracic Society Standards of Care Committee) and to members of the IBD Section (Chairman, Dr S Travis) and Clinical Services Committee (Chairman, Dr M Denyer) of the British Society of Gastroenterology for reviewing this paper and for their helpful suggestions.

Gut 2005;54:1360-1362.

doi: $10.1136 /$ gut.2005.076034

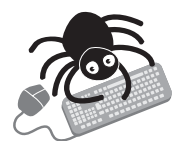

Conflict of interest: declared (the declaration can be viewed on the Gut website at http://www.gutjnl.com/ supplemental)

Correspondence to: Professor D S Rampton, Endoscopy Unit, Royal London Hospital, London E1 1BB, UK; d.rampton@qmul.ac.uk

\section{REFERENCES}

1 Ormerod LP, Milburn HJ, Gillespie S, et al. BTS recommendations for assessing risk, and for managing $M$ tuberculosis infection and disease in patients due to start anti-TNF alpha treatment. Thorax Published Online First: 29 July 2005. doi: $10.1136 /$ thx.2005.046797.

2 Rutgeerts P, van Assche G, Vermeire S. Optimizing anti-TNF treatment in inflammatory bowel disease. Gastroenterology 2004; 126:1593-610.

3 Maini SR. Infliximab treatment of rheumatoid arthritis. Rheum Dis Clinics N Am 2004;30:329-47.

4 Braun J, Sieper J. Biological therapies in the spondyloarthropathies - the current state. Rheumatology 2004;43:1072-84.

5 Rutgeerts P, Feagan BG, Olson A, et al. A randomized placebo-controlled trial of infliximab therapy for active ulcerative colitis: Act 1 trial. Gastroenterology 2005;128:A-105.

6 Sandborn WJ, Rachmilewitz D, Hanaver SB, et al. Infliximab induction and maintenance therapy for ulcerative colitis: the Act 2 trial. Gastroenterology 2005; 128:A-104.

7 Jarnerot G, Hertevig E, Friis-Liby I-L, et al. Infliximab as rescue therapy in severe to moderately severe ulcerative colitis. A randomised placebo-controlled study. Gastroenterology 2005; 128:1805-11.

8 Rose AMC, Watson JM, Graham C, et al. Tuberculosis at the end of the 20th century in England and Wales: results of a national survey in 1998. Thorax 2001;56:173-9.

9 Keane J, Gershon S, Wise RP, et al. Tuberculosis associated with infliximab, a tumor necrosis factor-alpha neutralizing agent. N Engl J Med 2001;345: 1098-104

10 Wallis RS, Broder MS, Wong JY, et al. Granulomatous infectious diseases associated with tumor necrosis factor antagonists. Clin Infect Dis 2004;38:1261-5.

11 Gomez-Reino JJ, Carmona L, Valverde VR, BIOBADASER Group, et al. Treatment of rheumatoid arthritis with tumour necrosis factor inhibitors may predispose to significant increase in tuberculosis risk: a multicenter activesurveillance report. Arthritis Rheum 2003;48:2122-7. 
12 Gardam MA, Keystone EC, Menzies R, et al. Antitumour necrosis factor agents and tuberculosis risk: mechanisms of action and clinical management. Lancet Inf Dis 2003;3:148-55.

13 Sandborn WJ, Hanaver SB. Infliximab in the treatment of Crohn's disease: a user's guide for clinicians. Am J Gastroenterol 2002:97:2962-72.
14 Mow WS, Abreu-Martin MT, Papadakis KA, et al. High incidence of anergy in inflammatory bowel disease patients limits the usefulness of PPD screening before infliximab therapy. Clin Gastroenterol Hepatol 2004;2:309-13.

15 European Agency for Evaluation of Medicinal Products (EMEA) Public Statement on Infliximab (Remicade): update on safety concerns (2002). http://www.emea.eu.int/pdfs/human/press/ pus/003202.pdf (accessed 16 August 2005).

16 NICE. Guidance on the use of infliximab for Crohn's disease. London: National Institute for Clinical Excellence, technology appraisal guidance, No 40, 2002 (also online at http://www.nice.org.uk/pdf/ NiceCROHNS40GUIDANCE.pdf; accessed 16 August 2005).

\section{Clinical Evidence - Call for contributors}

Clinical Evidence is a regularly updated evidence-based journal available worldwide both as a paper version and on the internet. Clinical Evidence needs to recruit a number of new contributors. Contributors are healthcare professionals or epidemiologists with experience in evidence-based medicine and the ability to write in a concise and structured way.

Areas for which we are currently seeking authors:

- Antenatal monitoring

- Dietary supplementation and treatments in pregnancy

- Palliative care

- Prolonged pregnancy

We are also looking for authors for existing topics. For full details on what these chapters are please visit www. clinicalevidence.com/ceweb/contribute/index.jsp

However, we are always looking for others, so do not let this list discourage you.

Being a contributor involves:

- Selecting from a validated, screened search (performed by in-house Information Specialists) epidemiologically sound studies for inclusion.

- Documenting your decisions about which studies to include on an inclusion and exclusion form, which we keep on file.

- Writing the text to a highly structured template (about 1500-3000 words), using evidence from the final studies chosen, within 8-10 weeks of receiving the literature search.

- Working with Clinical Evidence editors to ensure that the final text meets epidemiological and style standards.

- Updating the text every 6 months using any new, sound evidence that becomes available. The Clinical Evidence in-house team will conduct the searches for contributors; your task is simply to filter out high quality studies and incorporate them in the existing text.

- To expand the topic to include a new question about once every 12-18 months.

If you would like to become a contributor for Clinical Evidence or require more information about what this involves please send your contact details and a copy of your CV, clearly stating the clinical area you are interested in, to CECommissioning@bmigroup.com.

\section{Call for peer reviewers}

Clinical Evidence also needs to recruit a number of new peer reviewers specifically with an interest in the clinical areas stated above, and also others related to general practice. Peer reviewers are healthcare professionals or epidemiologists with experience in evidence-based medicine. As a peer reviewer you would be asked for your views on the clinical relevance, validity, and accessibility of specific topics within the journal, and their usefulness to the intended audience (international generalists and healthcare professionals, possibly with limited statistical knowledge). Topics are usually 1500-3000 words in length and we would ask you to review between 2-5 topics per year. The peer review process takes place throughout the year, and out turnaround time for each review is ideally 10-14 days.

If you are interested in becoming a peer reviewer for Clinical Evidence, please complete the peer review questionnaire at www. clinicalevidence.com/ceweb/contribute/peerreviewer.jsp 